\title{
Structure Properties of Koch Networks Based on Networks Dynamical Systems
}

\author{
Yinhu Zhai, ${ }^{1}$ Jia-Bao Liu, $^{2}$ and Shaohui Wang ${ }^{3}$ \\ ${ }^{1}$ School of Information Engineering, Guangdong University of Technology, Guangzhou 510006, China \\ ${ }^{2}$ School of Mathematics and Physics, Anhui Jianzhu University, Hefei 230601, China \\ ${ }^{3}$ Department of Mathematics and Computer Science, Adelphi University, Garden City, NY 11530, USA
}

Correspondence should be addressed to Jia-Bao Liu; liujiabaoad@163.com and Shaohui Wang; shaohuiwang@yahoo.com

Received 8 November 2016; Revised 20 January 2017; Accepted 12 February 2017; Published 6 March 2017

Academic Editor: Pietro De Lellis

Copyright (C) 2017 Yinhu Zhai et al. This is an open access article distributed under the Creative Commons Attribution License, which permits unrestricted use, distribution, and reproduction in any medium, provided the original work is properly cited.

\begin{abstract}
We introduce an informative labeling algorithm for the vertices of a family of Koch networks. Each label consists of two parts: the precise position and the time adding to Koch networks. The shortest path routing between any two vertices is determined only on the basis of their labels, and the routing is calculated only by few computations. The rigorous solutions of betweenness centrality for every node and edge are also derived by the help of their labels. Furthermore, the community structure in Koch networks is studied by the current and voltage characterizations of its resistor networks.
\end{abstract}

\section{Introduction}

The WS small-world models [1] and BA scale-free networks [2] are two famous random networks which stimulated an in-depth understanding of various physical mechanisms in empirical complex networks. The two main shortcomings are the uncertain creating mechanism and the huge computation in analysis. Deterministic models always have important properties similar to random models, such as being scalefree, having small-world behavior, and being highly clustered, and thus they could be used to imitate empirical networks appropriately. Hence, the study of the deterministic models of a complex network is increasing recently.

Inspired by the simple recursive operation and techniques of plane filling and generating processes of fractals, several deterministic models [3-18] have been created imaginatively and studied carefully. The lines in the famous Koch fractals [19] are mapped into vertices, and there is an edge between two vertices if two lines are connected; the generated novel networks were named Koch networks [20]. This novel class of networks incorporates some key properties which characterize the majority of real-life networked systems: a power-law distribution with exponent in the range between 2 and 3, a high clustering coefficient, a small diameter, and average path length and degree correlations. Besides, the exact numbers of spanning trees, spanning forests, and connected spanning subgraphs in the networks are enumerated by Zhang et al. in [20]. All these features are obtained exactly according to the proposed generation algorithm of the networks considered [21-29]. The deterministic models of the complex network have a fixed shortest path, but how to mark it only by their labels is rarely researched [30-34].

However, some important properties in Koch networks, such as vertex labeling, the shortest path routing algorithm, the length of the shortest path between arbitrary two vertices, the betweenness centrality, and the current and voltage properties of Koch resistor networks have not yet been researched. In this paper, we introduce an informative labeling and routing algorithm for Koch networks. By the intrinsic advantages of the labels, we calculate the shortest path distances between two arbitrary vertices in a couple of computations. We derive the rigorous solutions of betweenness centrality of every node and edge, and we also research the current and voltage characteristics of Koch resistor networks. 


\section{Koch Networks}

The Koch networks are constructed in an iterative way. Let $K_{m, t}$ be the Koch networks after $t \in N$ iterations, where $m$ is a structural parameter.

Definition 1. The Koch networks $K_{m, t}$ are generated as follows: initially $(t=0), K_{m, 0}$ is a triangle. For $t \geq 1, K_{m, t}$ is obtained from $K_{m, t-1}$ by adding $m$ groups of vertices to each of the three vertices of every existing triangle in $K_{m, t-1}$.

Remark 2. Each group consists of two new vertices, called son vertices. Both sons are connected to one another and to their father vertices; thus, the three vertices shape a new triangle.

That is to say, we can get $K_{m, t}$ from $K_{m, t-1}$ just by replacing each existing triangle in $K_{m, t-1}$ with the connected clusters on the right-hand side of Figure 1.

For the integrity of the article, we firstly introduce some important properties of Koch networks from [20]. The numbers of vertices and edges in networks $K_{m, t}$ are

$$
\begin{aligned}
& N_{t}=2(3 m+1)^{t}+1, \\
& E_{t}=3(3 m+1)^{t}
\end{aligned}
$$

By denoting $\Delta_{v}(t)$ as the numbers of nodes created at step $t$, we obtain $\Delta_{v}(t)=6 m(3 m+1)^{t-1}$; then, we also get that the degree distribution is $P\left(k=2(m+1)^{t-i}\right)=6 m(3 m+$ $1)^{i-1} /\left(2(3 m+1)^{t}+1\right)$; by substituting $i=t-\ln (k / 2) / \ln (m+1)$, for $a^{-\ln b / \ln c}=b^{-\ln a / \ln c}$, and then $(3 m+1)^{-\ln (k / 2) / \ln (m+1)}=$ $2^{\ln (3 m+1) / \ln (m+1)} k^{-\ln (3 m+1) / \ln (m+1)}$ and $P(k)=[6 m(3 m+$ $\left.1)^{t-1} / 2(3 m+1)^{t}+1\right] 2^{\ln (3 m+1) / \ln (m+1)} k^{-\ln (3 m+1) / \ln (m+1)}$, in the infinite $t$ limit, one can get

$$
P(k)=\frac{3 m}{3 m+1} 2^{\ln (3 m+1) / \ln (m+1)} k^{-\ln (3 m+1) / \ln (m+1)} .
$$

Then, the exponent of degree distribution is $\gamma=\ln (3 m+$ $1) / \ln (m+1)$, which belongs to the interval $(1,2]$. The average clustering coefficient $C$ of the whole network is given by $C=\left(1 / N_{t}\right) \sum_{r=0}^{t}\left(1 /\left(k_{i}(r)-1\right)\right) L_{u}(r)$. When $m$ increases from 1 to infinity, $C$ increases from 0.82008 to 1 . So, the Koch networks are highly clustered. The average path length (APL) approximates $4 m t /(3 m+1)$ in the infinite $t$, for APL is

$$
\begin{aligned}
d_{t} & =\frac{3 m+5+(24 m t+24 m+4)\left(3 m+1^{t}\right)}{3(3 m+1)\left[2(3 m+1)^{t}+1\right]} \\
& \sim \frac{4 m t}{3 m+1} .
\end{aligned}
$$

This formula shows that Koch networks exhibit smallworld behavior. These properties indicate that Koch networks incorporate some key properties characterizing a majority of empirical networks: simultaneously scale-free, small-world, and highly clustered [20].

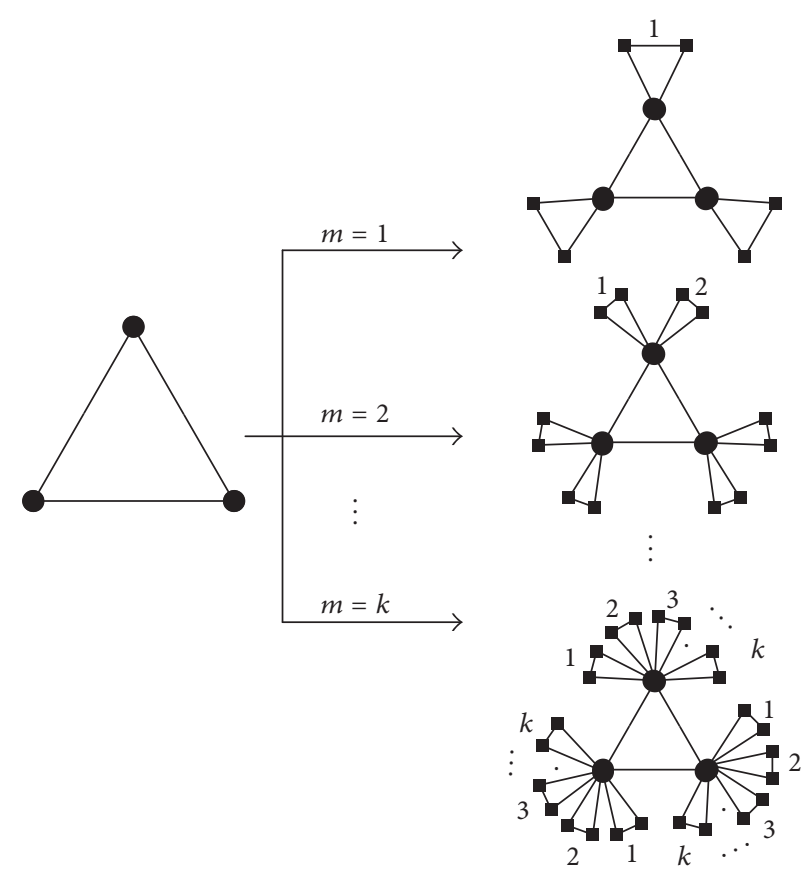

FIGURE 1: Iterative construction method for the Koch networks when $m=1,2, \ldots, k$.

\section{Vertex Labeling and Routing Algorithm by the Shortest Path}

Definition 3. All the vertices are located in three different subnetworks of the Koch network; the label $n$ ( $n=1,2$, or 3 ) is used to denote the subnetworks.

Remark 4. Denote the three symmetrical subnetworks in Koch networks $K_{m, t}$ as $K_{m, t}^{1}, K_{m, t}^{2}$, and $K_{m, t}^{3}$. Then, $K_{m, t}$ is obtained just by linking the hub of three subnetworks directly. Therefore, the label $n(n=1,2$, or 3$)$ is used to differentiate the vertices in the three different subnetworks $K_{m, t}^{n}$.

A binary digits code is used to identify the precise position of a vertex in $K_{m, t}^{n}$ and the exact time which is linked to $K_{m, t}^{n}$. The method is shown as follows.

Definition 5. Any vertex in $K_{m, t}^{n}$ is marked with binary digits $b_{1} b_{2} b_{3} \cdots b_{j}$, where $b_{1}=0$ when $j=1 . b_{j}=0$ or 1 when $j=$ $2,3, \ldots, t$. The 0 (or 1 ) in binary digits represents the notion that the new $m$ vertices are grown from a son vertex (or father vertex) in a triangle. The length of the binary digit is the time at which the vertex is linked to Koch networks.

Remark 6. Because the initial network $K_{m, 0}$ is a triangle, all the three initial vertices in it have no father vertices, so that the new vertices adding to the initial vertices should be marked with 0 at time $j=1$; that is, $b_{1}$ must be 0 .

Then, we obtain the set $S$, by processing the binary digit codes of each vertex in $K_{m, t}$.

$$
S=\left\{\Phi, b_{1}, b_{1} b_{2}, b_{1} b_{2} b_{3}, \ldots, b_{1} b_{2} b_{3} \cdots b_{t}\right\}
$$


Remark 7. The element $\Phi$ in $S$ implies that when $t=0$, the length of $\Phi$ is zero in $K_{m, 0}$.

Definition 5 implies that all the vertices, added to an existing vertex at step $j$, have the same binary codes $b_{1} b_{2} b_{3} \cdots b_{j}$. Consequently, the number of vertices which are added to an existing father vertex at step $j$ is given by

$$
l_{\max }(j)=(2 m)^{j-\sum_{i=1}^{j} b_{i}}(m+1)^{\sum_{i=1}^{j} b_{i}} .
$$

So we need to mark the vertices of this group with an extra integer $l(j) \in\left[1, l_{\max }(j)\right]$ for they all have the same binary codes $b_{1} b_{2} b_{3} \cdots b_{j}$ and the same group indicator $n$.

Definition 8. An integer $l(j)$ is used to identify the precise position, increasing by clockwise direction, of a vertex in the group which is added to a father vertex at the iteration $j$.

Remark 9. Because $l(j)$ increases from 1 and is positioned after the binary blue code, a dot is needed between $l(j)$ and the binary code to avoid confusion.

In sum, the arbitrary vertex which is added to $K_{m, t}$ at step $j$ is labeled with $n b_{1} b_{2} b_{3} \cdots b_{j} \cdot l(j)$. The code $n$ denotes which subnetwork of $K_{m, t}^{n}$ the vertex belongs to; the binary digit $b_{1} b_{2} b_{3} \cdots b_{j}$ indicates the father vertex which is linked to this new vertex; the positive integer $l$, which increases in a clockwise manner, is used to mark the precise position around a father vertex.

Define the set $M(j)$ as the label set of the vertices added to networks $K_{m, t}$ at step $j$. It is apparent that $M(0)=\{1,2,3\}$ and $M(j)=\left\{n b_{1} b_{2} b_{3} \cdots b_{j} \cdot l(j)\right\}$. Let the set $L_{m, t}$ represent the labels of all the vertices in $K_{m, t}$; we obtain

$$
L_{m, t}=\bigcup_{j=1}^{t} M(j) .
$$

For example, Figure 2 demonstrates the vertex labeling of all the vertices in Koch network $K_{2,2}$. In the following sections, we deduced some important properties of Koch networks just on the basis of the labels of their vertices.

Property 1. Each vertex has a unique label.

Suppose an arbitrary vertex is labeled with $n b_{1} b_{2} b_{3} \cdots b_{j}$. $l(j)$. Firstly, from the labeling algorithm, the labels of any pair of vertices are different from each other. Secondly, the size of $L_{m, t}$ equals the size of Koch networks. So, we deduced that any vertex has a unique label.

Assume that $n b_{1} b_{2} b_{3} \cdots b_{i} \cdot l_{v}(i)$ is the label of arbitrary vertex $v$ which is added to $K_{m, t}$ at step $i$, and let the set $A(v)$ denote the labels of all neighbor vertices of $v$. By comparing the vertex's degree between $v$ and its neighbors, $A(v)$ can be divided into three subsets: $A_{e}(v), A_{l}(v)$, and $A_{h}(v)$, the vertices in which sets have a degree equal to, lower than, and higher than the degree of $v$, respectively. That is to say, $A(v)=A_{e}(v) \cup A_{l}(v) \cup A_{h}(v)$.
Property 2. $A_{e}(v)=\left\{n b_{1} b_{2} b_{3} \cdots b_{i} \cdot l_{e}(i)\right\}$, where vertex degree $l_{e}(i)=l_{v}(i)+1$ if $\bmod \left(l_{v}(i), 2\right)=1$, or $l_{e}(i)=l_{v}(i)-1$ if $\bmod \left(l_{v}(i), 2\right)=0$.

According to the proposed algorithm, at each step, any father vertex adds $m$ groups of vertices, with each group consisting of two vertices. Thus, these latter, together with the father, are linked to each other and form a new triangle. Therefore, the two vertices in the same group are neighbors which are linking directly and have the same degrees. By the labeling method, the $m$ group vertices are labeled with the integers $l(i)$ which increase from 1 to $l_{\max }(i)$ in a clockwise manner. So, $n b_{1} b_{2} b_{3} \cdots b_{i} \cdot\left(l_{v}(i)+1\right)$ is the neighbor of $n b_{1} b_{2} b_{3} \cdots b_{i} \cdot l_{v}(i)$ with the same degrees if $\bmod \left(l_{v}(i), 2\right)=1$, or $n b_{1} b_{2} b_{3} \cdots b_{i} \cdot\left(l_{v}(i)-1\right)$ is the neighbor if $\bmod \left(l_{v}(i), 2\right)=0$.

Property 3. $A_{l}(v)=\left\{n b_{1} b_{2} b_{3} \cdots b_{i} 0 \cdot l_{v}(i+1), n b_{1} b_{2} b_{3} \cdots b_{i} 0\right.$. $\left.l_{v}(i+2), \ldots, n b_{1} b_{2} b_{3} \cdots b_{i} 0 \cdot l_{v}(t)\right\}$.

From the labeling algorithm, the vertices with longer binary codes have lower degrees than the vertices with shorter binary codes. In addition, the 0 or 1 in binary codes indicates that the new vertex is growing from the two son vertices or father vertex in each triangle. Hence, we can understand that the vertices, adding to $v$ at steps $i+1, i+2, \ldots, t$, are labeled with $n b_{1} b_{2} b_{3} \cdots b_{i} 0 \cdot l_{v}(i+1), n b_{1} b_{2} b_{3} \cdots b_{i} 0 \cdot l_{v}(i+$ $2), \ldots, n b_{1} b_{2} b_{3} \cdots b_{i} 0 \cdot l_{v}(t)$.

Let $\lceil x\rceil$ be the function returning the biggest integer just smaller than real number $x$.

Property 4. $A_{h}(v)=\left\{n b_{1} b_{2} b_{3} \cdots b_{j-1} \cdot\left\lceil l_{v}(i) / 2 m(m+\right.\right.$ 1) $\left.\left.\sum_{k=j+1}^{i} b_{k}\right\rceil\right\}$.

We remark that $n b_{1} b_{2} b_{3} \cdots b_{i} \cdot l_{v}(i)$ is the label of an arbitrary vertex $v$. From construction, we obtain that the label of the only father vertex of $v$ depends on the composition in binary codes $b_{1} b_{2} b_{3} \cdots b_{i}$ of vertex $v$. Suppose the first 0 in $b_{1} b_{2} b_{3} \cdots b_{i}$, from the right to the left side, is $b_{j}$. By the construction method, it is clear that $v$ is linked to a vertex with higher degree which is labeled with $n b_{1} b_{2} b_{3} \cdots b_{j-1}$. $\left\lceil l_{v}(i) / 2 m(m+1)^{\sum_{k=j+1}^{i} b_{k}}\right\rceil$. In particular, if the first 0 of $b_{1} b_{2} b_{3} \cdots b_{i}$ is $b_{1}$, the vertex with a higher degree is exactly a hub of Koch networks which is labeled with $n=1,2$ or 3 .

The deterministic models of the complex network have a fixed shortest path, but how to mark it only by their labels is rarely researched [15]. The following rules are used to determine the shortest path routing between any two vertices by the help of their labels. Let $n b_{1} b_{2} b_{3} \cdots b_{i} \cdot l$ and $n^{\prime} b_{1}^{\prime} b_{2}^{\prime} b_{3}^{\prime} \cdots b_{j}^{\prime}$. $l^{\prime}$ be the labels of arbitrary pair of vertices in $K_{m, t}$.

Algorithm 10. The shortest path routing algorithm in Koch networks is discussed.

If $n \neq n^{\prime}$, find out, by Property 4, all the higher degree neighbors of the two vertices, until the hubs $n$ and $n^{\prime}$. Thus, by linking all vertices of them, we obtained the shortest path.

If $n=n^{\prime}$, the first step is to mark higher degree neighbors until the common highest degree vertex by Property 4 and then judge whether the two second highest degree vertices 


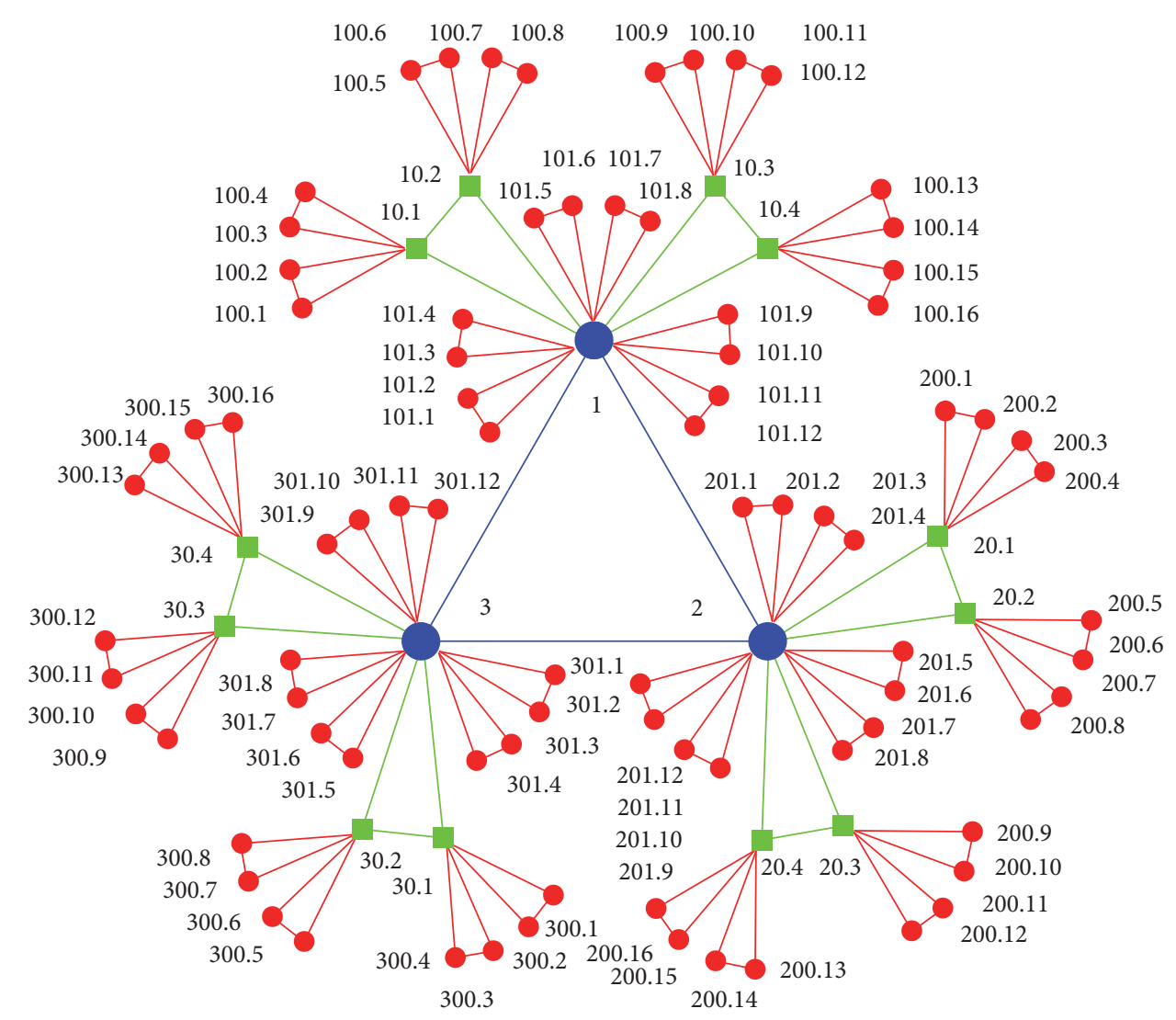

FIGURE 2: The labeling of Koch network $K_{2,2}$ when $m=2$ and $t=0,1,2$. The blue dots are initial vertices; the green squares are vertices adding at $t=1$; the red dots denote vertices adding to the network at $t=2$.

are neighbors or not by Property 3; if not, the shortest path is connected to all higher degree neighbors until the highest degree vertex; if so, the shortest path is just the same as above, only eliminating the highest degree vertex.

If $n \neq n^{\prime}$, the two vertices are located in different subnetworks $K_{m, n}^{n}$ and $K_{m, n}^{n^{\prime}}$. The routing by the shortest path between two vertices in different subsets is ascertained as follows. First, we obtain the neighbors which have higher degrees recursively by Property 4, until the hubs $n$ and $n^{\prime}$. Then, we connect all of them in turn; this is the only shortest path between two vertices.

If $n=n^{\prime}$, it is clear that the shortest path is located in the same subnetworks $K_{m, t}^{n}$. We find out the neighbors with higher degree by using Property 4 repeatedly, until the common highest degree vertex. Then, we judge whether the two second highest degree vertices are neighbors or not by Property 3. If they are not neighbors, we determine the shortest path as above by linking all the higher degree vertices until the highest vertex, by the help of the construction method of Koch networks. Else, if they are neighbors, the shortest path is the same as above by excluding the highest degree vertex.

The shortest path between any pair of vertices in $K_{m, t}$ is obtained after no more than $2 t$ times of integral computations and modulo operations by the help of the labeling method and routing algorithm proposed in this research. That is to say, the shortest path routing and the shortest distance between an arbitrary pair of vertices in Koch networks can be dealt with in few computations.

\section{Betweenness Centrality}

Betweenness centrality is originated from the analysis of the importance of the individual in social networks, including the betweenness of any vertex and edge in networks. If the betweenness of a node/edge is bigger, then the node/edge in the social network is more important [2]. The betweenness of a vertex for undirected networks is given by the expression

$$
g(v)=\frac{\sum_{s \neq v \neq t} \sigma_{s t}(v)}{\left(N_{t}-1\right)\left(N_{t}-2\right) / 2},
$$

where $\sigma_{s t}(v)$ is the number of the shortest paths passing through $v$. The computation of betweenness is very difficult in most networks. Fortunately, the betweenness of Koch networks can be derived qualitatively and quantitatively by the help of their labels in Koch networks, which is shown as follows.

Suppose that an arbitrary vertex $v$, which is adding to $K_{m, t}$ at time $i$, is labeled with $n b_{1} b_{2} b_{3} \cdots b_{i} \cdot l_{v}(i)$. The vertices in $K_{m, t}$ can be divided into three parts: the vertex $v$, the offspring vertices which are connected to $v$ directly and indirectly after 
step $i$ (they all have lower degrees than $v$ ), and the other vertices in $K_{m, t}$. Assume that the number of the second part vertices is $N_{l}$, and it can be worked out that $N_{l}=(2(3 m+$ $\left.1)^{t-i}-2\right) / 3$ by (1). Apparently, the number of the third parts is $N_{t}-N_{l}-1$. For the shortest path routing between any two vertices is unique, we get that $\sum_{s \neq v \neq l} \sigma_{s t}(v)=N_{l}\left(N_{t}-N_{l}-1\right) / 2$. Substitute this equation and (1) into (8); the betweenness of a vertex which is labeled with $n b_{1} b_{2} b_{3} \cdots b_{i} \cdot l_{v}(i)$ is given by

$$
\begin{aligned}
& g(v) \\
& =\frac{2\left[(3 m+1)^{t-i}-1\right]\left[3(3 m+1)^{t}-(3 m+1)^{t-i}-1\right]}{3(3 m+1)^{t}\left[2(3 m+1)^{t}-1\right]} .
\end{aligned}
$$

For $i=t-\ln (k / 2) / \ln (m+1)$ and $a^{-\ln (b) / \ln (c)}=b^{-\ln (a) / \ln (c)}$, we obtain the formula $g(v) \sim c_{1} k^{\ln (3 m+1) / \ln (m+1)}$ which holds with $c_{1}>0$. Therefore, the vertex betweenness in Koch networks is exponentially proportional to the vertex's degree with an exponent $\gamma=\ln (3 m+1) / \ln (m+1)$ belonging to the interval $(1,2]$.

The betweenness of edges can also be deduced in a similar way. Note $e$ as the edge between any two neighbor vertices $v$ and $u$ which are labeled with $n b_{1} b_{2} b_{3} \cdots b_{i} \cdot l_{v}(i)$ and $n^{\prime} b_{1}^{\prime} b_{2}^{\prime} \cdots b_{j}^{\prime} \cdot l_{u}^{\prime}(j)$. Without loss of generality, assume that vertex $u$ has higher degree than $v$. So the label of $u$ belongs to the set $A_{h}(v)$ by Property 4 . Suppose a triangle is shaped by three vertices: $v, u$, and $w$. Therefore, $w$ has the same degree as $v$. Then, Koch network $K_{m, t}$ can be divided into three parts: the lower degree vertices linking to $v$ directly or indirectly, the vertices connected to $u$ directly or indirectly, and the lower degree vertices adding directly or indirectly, respectively. Correspondingly, the label set $L_{m, t}$ falls into three subsets: $A_{e l}(v), A_{\text {other }}(u)$, and $A_{e l}(w)$. The relationship of these four label sets is shown as follows:

$$
L_{m, t}=A_{e l}(v) \cup A_{\text {other }}(u) \cup A_{e l}(w) .
$$

The size of $A_{e l}(v)$ is derived as $N_{e l}(v)=\left(2(3 m+1)^{t-i}+\right.$ $1) / 3$. From the symmetry of vertices $v$ and $w$, we obtain $N_{e l}(v)=N_{e l}(w)$. Then, we get that

$$
\begin{aligned}
N_{\text {other }}(u) & =N_{t}-N_{e l}(v)-N_{e l}(w) \\
& =2(3 m+1)^{t-i}+1-\frac{2(3 m+1)^{t-i}+1}{3}
\end{aligned}
$$

from the construction of Koch networks. For the shortest path between any two vertices is unique, then the betweenness of the edge $e$ is defined as follows:

$$
g(e)=\frac{N_{\mathrm{el}}(v) N_{\mathrm{other}}(u) / 2}{\left(N_{t}-1\right)\left(N_{t}-2\right) / 2} .
$$

Therefore, the betweenness centrality of the edge $e$ is given by

$$
\begin{aligned}
& g(e) \\
& =\frac{\left[2(3 m+1)^{t-i}+1\right]\left[6(3 m+1)^{t}-4(3 m+1)^{t-i}+1\right]}{18(3 m+1)^{t}\left[2(3 m+1)^{t}-1\right]} .
\end{aligned}
$$

Therefore, the edge betweenness holds $g(e)$ $c_{2} k^{\ln (3 m+1) / \ln (m+1)}$, where $c_{2}>0$. The edge betweenness is also exponentially proportional to the degree of the lower degree vertex $v$, and the exponent is $\gamma=\ln (3 m+1) / \ln (m+1)$ belonging to the interval $(1,2]$. In a word, the betweenness of an edge is exponentially proportional to the time of adding to Koch networks.

\section{Resistor Networks}

The communities in networks are the groups of vertices within which the connections are dense but between which the connections are sparser. A community detection algorithm which is based on voltage differences in resistor networks is described in $[35,36]$. The electrical circuit is formed by placing a unit resistor on each edge of the network and then applying a unit potential difference (voltage) between two vertices chosen arbitrarily. If the network is divided strongly into two communities and the vertices in question happen to fall in different communities, then the spectrum of voltages on the rest of the vertices should show a large gap corresponding to the border between the communities. For more work on resistance distance and resistor networks, the readers are referred to the recent papers $[37,38]$.

Moreover, the information in complex networks not always flows in the shortest path, so that the evaluation of the betweenness of nodes can also have other principles, such as the current-flow betweenness. Consider an electrical circuit created by placing a unit resistor on every edge of the network. One unit of current is injected into the network at a source vertex and one unit is extracted at a target vertex, so that the current in the network as a whole is conserved. Then, the current-flow betweenness of a vertex is defined as the amount of current that flows through in this setup; the average of the current flow over all source-target pairs is shown as follows:

$$
g_{v}=\frac{\sum_{s<t} I_{s t}(v)}{N_{t}\left(N_{t}-1\right) / 2},
$$

where $I_{s t}(v)$ is the current over vertex $v$.

After placing a unit resistor on every edge in $K_{m, t}$, insert one unit of current or voltage at source vertex $v_{0}$ labeled with $n b_{1} b_{2} b_{3} \cdots b_{i} \cdot l$, and further choose the target vertex $v_{m+1}$ with labels $n^{\prime} b_{1}^{\prime} b_{2}^{\prime} \cdots b_{j}^{\prime} \cdot l^{\prime}$. Assume the shortest path is from $v_{0}$ to vertices $v_{1}, \ldots, v_{m}$ until $v_{m+1}$. Therefore, the shortest distance is $m+1$. The property of Koch resister networks is described as follows.

Property 5. The voltages of vertices $\left\{v_{i}\right\}$ shape an arithmetic progression from 1 to 0 , and the step length is $1 /(m+1)$. The voltage of vertices $\left\{\overline{v_{k}}\right\}$ decreases from $1-1 /(2 m+2)$ to $1 /(2 m+$ $2)$, but the step length is also $1 /(m+1)$.

If $n \neq n^{\prime}$, from Algorithm 10, there are two hubs $v_{j}=n$ and $v_{j+1}=n^{\prime}$ with the highest degree in the shortest path. Hence, the vertices which are affected by unit voltage are $\left\{v_{i}\right\} \cup$ $\left\{\overline{v_{k}}\right\}$, where $i=0,1,2, \ldots, m+1, k=0,1,2, \ldots, j-1, j+$ $1, \ldots, m+1, \overline{v_{i}}$ is the neighbor of $v_{i}$ with the same degree, and 
apparently $\overline{v_{j+1}}$ is the other hub. The edges between vertices $\left\{v_{i}\right\} \cup\left\{\overline{v_{k}}\right\}$ formed $m+1$ triangles which are in series and the common vertices are $\left\{v_{i}\right\}$, so that the unit current only passes through these edges in whole Koch networks $K_{m, t}$.

If $n=n^{\prime}$ and there are two highest degree vertices, noting $v_{j}$ and $v_{j+1}$, in the shortest path, hence the unit voltage can only affect vertices $\left\{v_{i}\right\} \cup\left\{\overline{v_{k}}\right\}$ in $K_{m, t}$, where $i=0,1,2, \ldots, m+$ $1, k=0,1,2, \ldots, j-1, j+1, \ldots, m+1$, and $\overline{v_{i}}$ is the neighbor of $v_{i}$ with the same degree too, but $\overline{v_{j+1}}$ is a higher degree neighbor which is linked with $v_{j}$ and $v_{j+1}$ directly; the unit current also flows through the edges in $m+1$ triangles which are in series.

If $n=n^{\prime}$ but there are the only highest degree vertices, denoting $v_{j}$, in the shortest path, the unit voltage impacts vertices $\left\{v_{i}\right\} \cup\left\{\overline{v_{k}}\right\}$, where $i=0,1,2, \ldots, m+1, k=$ $0,1,2, \ldots, j-1, j+1, \ldots, m+1$, and $\overline{v_{i}}$ is the neighbor of $v_{i}$ with the same degree; the behavior of unit current is the same as the two conditions above.

Property 6. The current stream from the edges which are linked to the vertices $\left\{v_{i}\right\}$ is $2 / 3$, while the current passing though the edges linking to $\left\{\overline{v_{k}}\right\}$ is the remaining $1 / 3$.

The property can be proved similarly as the proof of Property 5 by the help of the forming mechanism of Koch resistor networks.

In brief, the spectrum of voltages on the vertices shows that Koch networks have no significant community structure in spite of having massive triangles between nodes. Also, the current flow can gauge well the importance of edges betweenness in Koch networks in information flowing which is not flowing only by the shortest path.

\section{Conclusions}

The family of Koch networks, with high clustering coefficient, scale-free, small diameter and average path length, and smallworld properties, successfully reproduces some remarkable characteristics in many nature and man-made networks and has special advantages in the research of some physical mechanisms such as random walk in complex networks.

We provided an informative vertex labeling method and produced a routing algorithm for Koch networks. The labels include full information about any vertices precise position and the time adding to the networks. By the help of labels, we marked the shortest path routing and the shortest distance between any pair of vertices in Koch networks. The needed computation is just no more than $2 t$ times of integral computations and modulo operations. Moreover, we derived the rigorous solution of betweenness centrality of every vertex and edge in Koch networks, and we also researched the current and voltage characteristics in Koch networks on the basis of their labels.

By the help of our results, in contrast with more usually probabilistic approaches, the deterministic Koch models will have unique virtues in understanding the underlying mechanisms between dynamical processes (random walk, consensus, stabilization, synchronization, etc.) to the structure of complex networks by the new method of rigorous derivation.

\section{Competing Interests}

The authors declare that there are no competing interests regarding the publication of this paper.

\section{Acknowledgments}

This work was supported by the Natural Science Foundation of Guangdong Province under Grant no. 2016A030313703, Guangdong Science and Technology Plan under Grant no. 2016B030305002, the National Natural Science Foundation of China under Grants nos. 61471130 and 61473093, and the Project of Anhui Jianzhu University under Grant no. 2016QD116.

\section{References}

[1] D. J. Watts and S. H. Strogatz, "Collective dynamics of 'smallworld' networks," Nature, vol. 393, no. 6684, pp. 440-442, 1998.

[2] A.-L. Barabási and R. Albert, "Emergence of scaling in random networks," Science, vol. 286, no. 5439, pp. 509-512, 1999.

[3] F. Comellas, J. Ozón, and J. G. Peters, "Deterministic smallworld communication networks," Information Processing Letters, vol. 76, no. 1-2, pp. 83-90, 2000.

[4] A.-L. Barabási, E. Ravasz, and T. Vicsek, "Deterministic scalefree networks," Physica A: Statistical Mechanics and its Applications, vol. 299, no. 3-4, pp. 559-564, 2001.

[5] S. Jung, S. Kim, and B. Kahng, "Geometric fractal growth model for scale-free networks," Physical Review E, vol. 65, no. 5, Article ID 056101, 2002.

[6] G. Corso, "Families and clustering in a natural numbers network," Physical Review E, vol. 69, no. 3, Article ID 036106, 2004.

[7] A. K. Chandra and S. Dasgupta, "A small world network of prime numbers," Physica A. Statistical Mechanics and its Applications, vol. 357, no. 3-4, pp. 436-446, 2005.

[8] K. Iguchi and H. Yamada, "Exactly solvable scale-free network model," Physical Review E, vol. 71, no. 3, Article ID 036144, 2005.

[9] J. P. Doye and C. P. Massen, "Self-similar disk packings as model spatial scale-free networks," Physical Review E. Statistical, Nonlinear, and Soft Matter Physics, vol. 71, no. 1, 2005.

[10] J. S. Andrade, H. J. Herrmann, R. F. Andrade, and L. R. Da Silva, "Apollonian networks: simultaneously scale-free, small world, euclidean, space filling, and with matching graphs," Physical Review Letters, vol. 94, no. 1, Article ID 018702, 2005.

[11] W. Xiao and B. Parhami, "Cayley graphs as models of deterministic small-world networks," Information Processing Letters, vol. 97, no. 3, pp. 115-117, 2006.

[12] T. Zhou, B.-H. Wang, P. M. Hui, and K. P. Chan, “Topological properties of integer networks," Physica A: Statistical Mechanics and Its Applications, vol. 367, pp. 613-618, 2006.

[13] Z.-Z. Zhang, S.-G. Zhou, and T. Zou, "Self-similarity, smallworld, scale-free scaling, disassortativity, and robustness in hierarchical lattices," European Physical Journal B, vol. 56, no. 3, pp. 259-271, 2007. 
[14] S. Boettcher, B. Gonçalves, and H. Guclu, "Hierarchical regular small-world networks," Journal of Physics. A. Mathematical and Theoretical, vol. 41, no. 25, 2008.

[15] F. Comellas and A. Miralles, "Modeling complex networks with self-similar outerplanar unclustered graphs," Physica A. Statistical Mechanics and its Applications, vol. 388, no. 11, pp. 2227-2233, 2009.

[16] S. Wang and B. Wei, "Multiplicative Zagreb indices of k-trees," Discrete Applied Mathematics, vol. 180, pp. 168-175, 2015.

[17] J.-B. Liu, C. Wang, S. Wang, and B. Wei, "Zagreb indices and multiplicative zagreb indices of eulerian graphs," Bulletin of the Malaysian Mathematical Sciences Society, 2017.

[18] J. Liu, S. Wang, C. Wang, and S. Hayat, "Further results on computation of topological indices of certain networks," IET Control Theory \& Applications, 2017.

[19] H. von Koch, "Une mthode gomtrique lmentaire pour ltude de certaines questions de la thorie descourbes planes," Acta Mathematica, vol. 30, no. 1, pp. 145-174, 1906.

[20] Z. Zhang, S. Gao, L. Chen, S. Zhou, H. Zhang, and J. Guan, "Mapping Koch curves into scale-free small-world networks," Journal of Physics. A. Mathematical and Theoretical, vol. 43, no. 39, Article ID 395101, 2010.

[21] D. Wei, X. Deng, X. Zhang, Y. Deng, and S. Mahadevan, "Identifying influential nodes in weighted networks based on evidence theory," Physica A: Statistical Mechanics and its Applications, vol. 392, no. 10, pp. 2564-2575, 2013.

[22] D.-J. Wei, Q. Liu, H.-X. Zhang, Y. Hu, Y. Deng, and S. Mahadevan, "Box-covering algorithm for fractal dimension of weighted networks," Scientific Reports, vol. 3, article 3049, 2013.

[23] D. Wei, B. Wei, H. Zhang, C. Gao, and Y. Deng, "A generalized volume dimension of complex networks," Journal of Statistical Mechanics: Theory and Experiment, vol. 2014, no. 10, Article ID P10039, 2014.

[24] Z. Zhang, S. Gao, and W. Xie, "Impact of degree heterogeneity on the behavior of trapping in Koch networks," Chaos, vol. 20, no. 4, Article ID 043112, 2010.

[25] Z.-G. Yu, H. Zhang, D.-W. Huang, Y. Lin, and V. Anh, "Multifractality and Laplace spectrum of horizontal visibility graphs constructed from fractional Brownian motions," Journal of Statistical Mechanics: Theory and Experiment, vol. 2016, Article ID 033206, 2016.

[26] Y.-Q. Song, J.-L. Liu, Z.-G. Yu, and B.-G. Li, "Multifractal analysis of weighted networks by a modified sandbox algorithm," Scientific Reports, vol. 5, article 17628, 2015.

[27] J.-L. Liu, Z.-G. Yu, and V. Anh, "A generalized volume dimension of complex networks. Determination of multifractal dimensions of complex networks by means of the sandbox algorithm," Chaos, vol. 25, no. 2, Article ID 023103, 2015.

[28] B. Li, Z. Yu, and Y. Zhou, "Fractal and multifractal properties of a family of fractal networks," Journal of Statistical Mechanics: Theory and Experiment, vol. 2014, no. 2, Article ID P02020, 2014.

[29] Y.-W. Zhou, J.-L. Liu, Z.-G. Yu, Z.-Q. Zhao, and V. Anh, "Multifractal and complex network analysis of protein dynamics," Physica A: Statistical Mechanics and Its Applications, vol. 416, pp. 21-32, 2014.

[30] Z. Zhang, F. Comellas, G. Fertin, A. Raspaud, L. Rong, and S. Zhou, "Vertex labeling and routing in expanded Apollonian networks," Journal of Physics. A. Mathematical and Theoretical, vol. 41, no. 3, 2008.

[31] F. Comellas and A. Miralles, "Vertex labeling and routing in self-similar outerplanar unclustered graphs modeling complex networks," Journal of Physics. A. Mathematical and Theoretical, vol. 42, no. 42, 2009.

[32] F. Comellas and A. Miralles, "Label-based routing for a family of scale-free, modular, planar and unclustered graphs," Journal of Physics A: Mathematical and Theoretical, vol. 44, no. 20, Article ID 205102, 2011.

[33] F. Comellas, G. Fertin, and A. Raspaud, "Vertex labeling and routing in recursive clique-trees, a new family of small-world scale-free graphs," in Proceedings of the 10th International Colloquium on Structural Information \& Communication Complexity (SIROCCO '03), Umea, Sweden, June 2003.

[34] Y. Zhai and Y. Wang, "Label-based routing for a family of smallworld Farey graphs," Scientific Reports, vol. 6, article 25621, 2016.

[35] M. E. J. Newman, "Detecting community structure in networks," European Physical Journal B, vol. 38, no. 2, pp. 321-330, 2004.

[36] M. E. J. Newman, "A measure of betweenness centrality based on random walks," Social Networks, vol. 27, no. 1, pp. 39-54, 2005.

[37] J.-B. Liu and J. Cao, "The resistance distances of electrical networks based on Laplacian generalized inverse," Neurocomputing, vol. 167, pp. 306-313, 2015.

[38] J.-B. Liu, W.-R. Wang, Y.-M. Zhang, and X.-F. Pan, "On degree resistance distance of cacti," Discrete Applied Mathematics. The Journal of Combinatorial Algorithms, Informatics and Computational Sciences, vol. 203, pp. 217-225, 2016. 


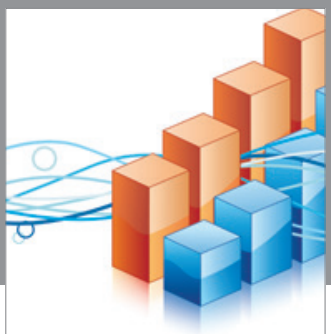

Advances in

Operations Research

vatem alat4

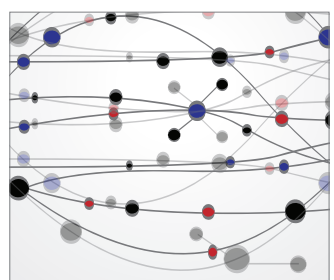

\section{The Scientific} World Journal
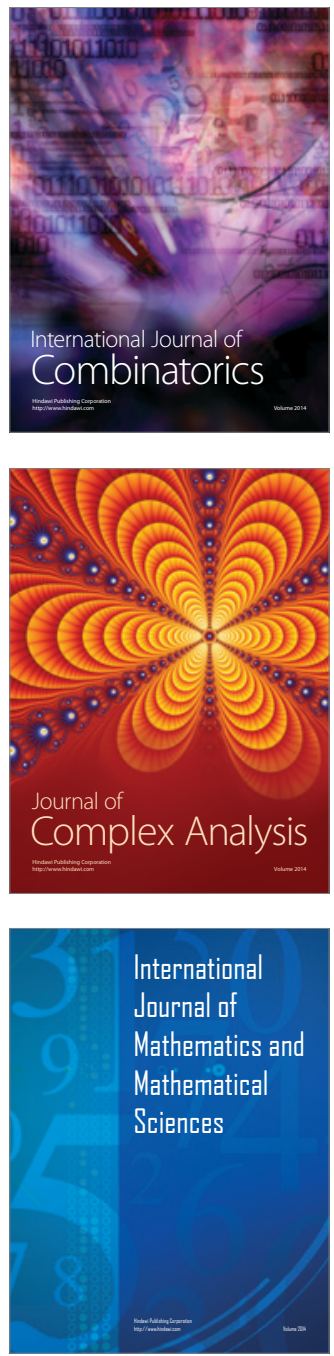
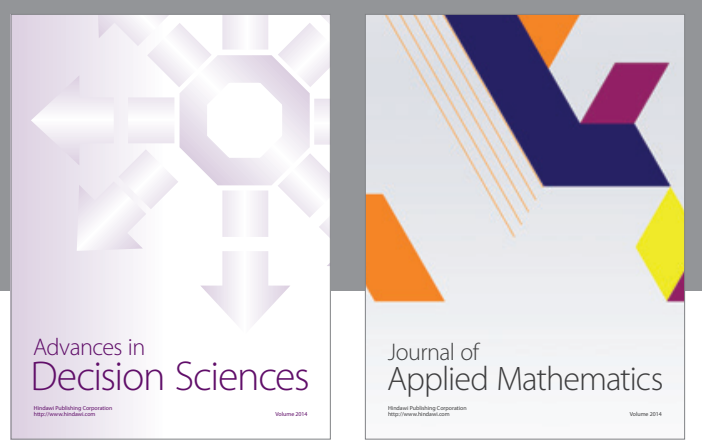

Algebra

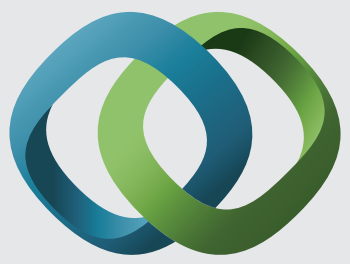

\section{Hindawi}

Submit your manuscripts at

https://www.hindawi.com
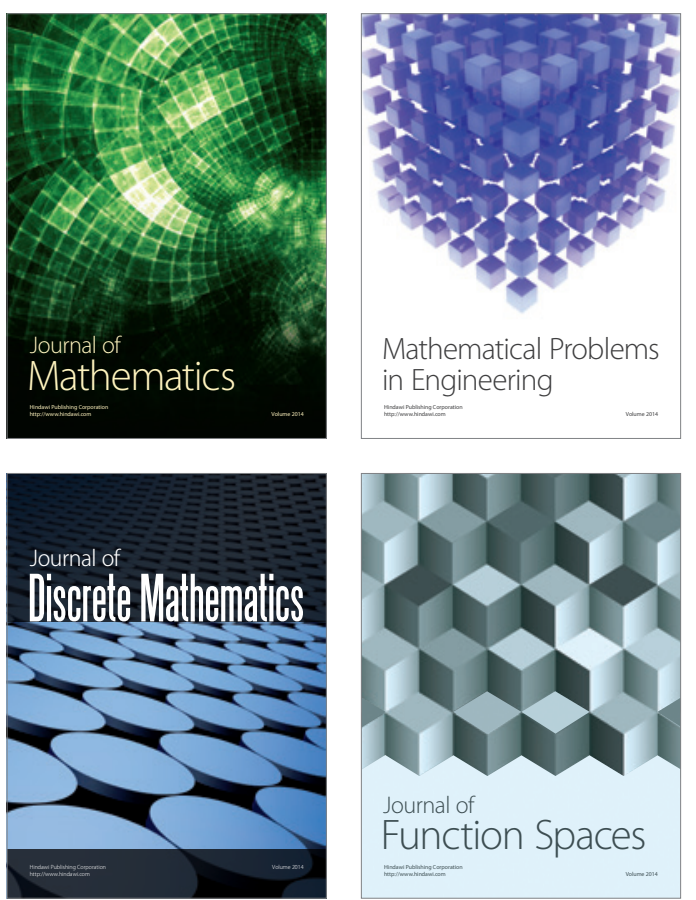

Mathematical Problems in Engineering
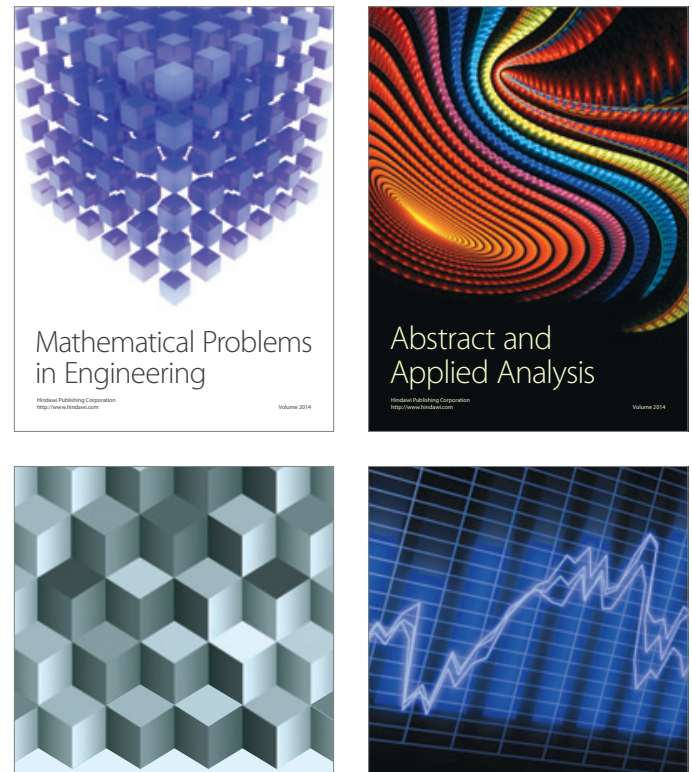

Journal of

Function Spaces

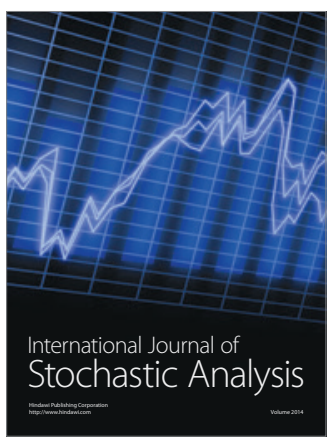

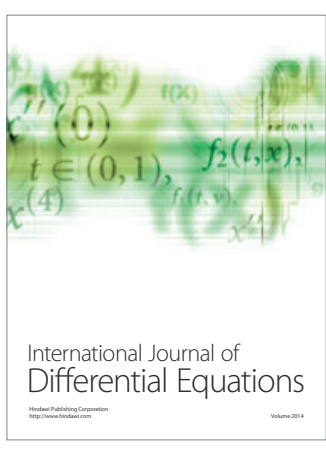
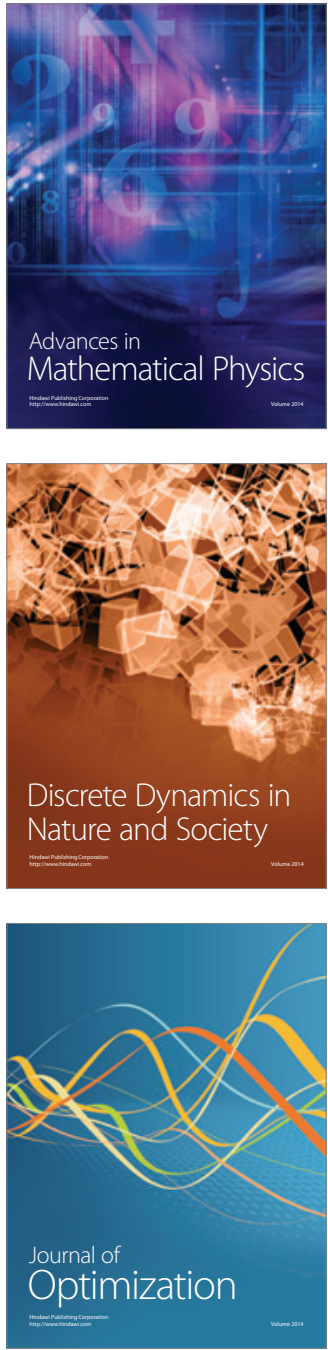